\title{
Modelagem Matemática da Dinâmica Veicular
}

\author{
Ana Paula Brezolin, Marcia de Fatima Brondani, Mauri Klein, Marnei Zorzella, \\ Scheila Cristiane Angnes Willers \\ Mestrandos em Modelagem Matemática - UNIJUÍ \\ 98700-000, Ijuí, RS \\ E-mail: anabrezolin@hotmail.com \\ marciabrondani@yahoo.com.br \\ mauri.klein@unijui.edu.br \\ marnei.zorzella@hotmail.com \\ scheila.willers@hotmail.com

\section{Antônio Carlos Valdiero} \\ Departamento de Ciências Exatas e Engenharias (DCEEng), UNIJUÍ 98280-000, Panambi, RS \\ E-mail: valdiero@unijui.edu.br
}

\begin{abstract}
Resumo: Este estudo expõe a modelagem matemática da dinâmica veicular considerando a força de atrito de arraste. A força de atrito de arraste exibe características não lineares devido ao escoamento turbulento do ar em torno do veículo, tornando o sistema dinâmico mais complexo. Na abordagem, apresenta-se uma descrição sobre as peculiaridades não lineares do atrito de arraste, enfatizando como o mesmo intervém no movimento de um veículo. Após, é proposto o modelo matemático para representar o comportamento dinâmico do sistema não linear que representa o comportamento da posição do veículo. Determina-se o ponto de equilíbrio e faz-se a análise da estabilidade. Assim, é realizada a simulação computacional e o resultado é representado através do plano de fase, possibilitando observar os efeitos não lineares presentes no estudo qualitativo do referido sistema.
\end{abstract}

Palavras-chave: Modelagem matemática, atrito de arraste, não linearidade, dinâmica veicular.

\section{Introdução}

Os sistemas dinâmicos são caracterizados pela variação de algumas grandezas em função do tempo. Comumente, estudos teóricos são realizados sobre determinadas situações com a finalidade de conhecer e compreender como tais conjunturas se organizam em sistemas. A significância desse conhecimento pode ser confirmada no exemplo citado por Monteiro (2002), o qual destaca que um sistema de pilotagem de avião precisa ser submetido a análises teóricas e a testes de simuladores de vôo para que possa suportar condições de turbulência. Assim, através de equações faz-se a representação dos comportamentos dinâmicos do sistema.

Nesse contexto, o objetivo desse trabalho é abordar a modelagem matemática e a simulação computacional da dinâmica da posição de um veículo, considerando o atrito de arraste. Para delinear esse estudo, primeiramente apresenta-se uma descrição bibliográfica sobre as características não lineares do atrito de arraste presentes no sistema. Continuando, expõe-se o modelo matemático para representar o comportamento dinâmico do sistema não linear do atrito veicular, determinando a solução de equilíbrio e as condições de estabilidade, seguido dos resultados obtidos na simulação computacional.

A formulação de modelos de sistemas dinâmicos não lineares exige processos matemáticos mais complexos para obter a descrição do comportamento do sistema, aplicados em várias áreas interdisciplinares. Então, evidencia-se que a modelagem matemática auxilia para fins de simulação e de análise de comportamentos dinâmicos, permitindo testar estratégias e prever futuros problemas através de informações e estimativas oriundas de especificações de componentes do sistema dinâmico, objeto de estudo.

Nos sistemas não lineares, como a modelagem matemática é normalmente proposta por meio de equações diferenciais, uma pequena variação nos parâmetros pode ocasionar o início de uma 
alteração no comportamento dinâmico do sistema. Tais fenômenos acontecem devido às características não lineares do sistema. Dentre essas características, destaca-se a não linearidade do atrito presente no sistema dinâmico da posição veicular.

Para Valdiero (2005), o atrito é definido como um fenômeno não linear multifacetado que exibe diversas características não lineares. De acordo com o autor, essas características são compostas pelo atrito estático, atrito de Coulomb, atrito viscoso e de arraste, que compõem os modelos mais simples baseados em mapas estáticos e por fenômenos dinâmicos mais complexos, como atrito de Stribeck, atrito estático crescente, memória de atrito e deslocamento de predeslizamento.

Nesse sentido, será dado enfoque as particularidades do atrito de arraste. Miotto (2009) baseando-se em GE et al. (2001), aborda que o atrito de arraste refere-se ao atrito causado pela resistência ao movimento de um corpo através de um fluido, como a resistência do ar. Ainda, enfatiza que ele é proporcional ao quadrado da velocidade e muitas vezes, decorre de um escoamento turbulento.

O resultado da interação da corrente de ar com o veículo em movimento gera uma componente na direção axial do móvel, que se opõem ao movimento do mesmo, intervindo no seu comportamento dinâmico. Esse tipo de atrito é pertinente com as tensões de cisalhamento atuantes e as superfícies em contato, as quais podem ser lisas ou ásperas. Conforme vários estudos relacionados, verifica-se que em superfícies mais lisas o ar se move de forma laminar, porém em superfícies ásperas, ocorre um fluxo de ar turbilhonado aumentando o arrasto.

Então, para melhor entender como o atrito de arraste interfere na posição veicular, torna-se oportuno abordar detalhadamente o desempenho do ar em contato com uma superfície, citado por Leal et al (2012).

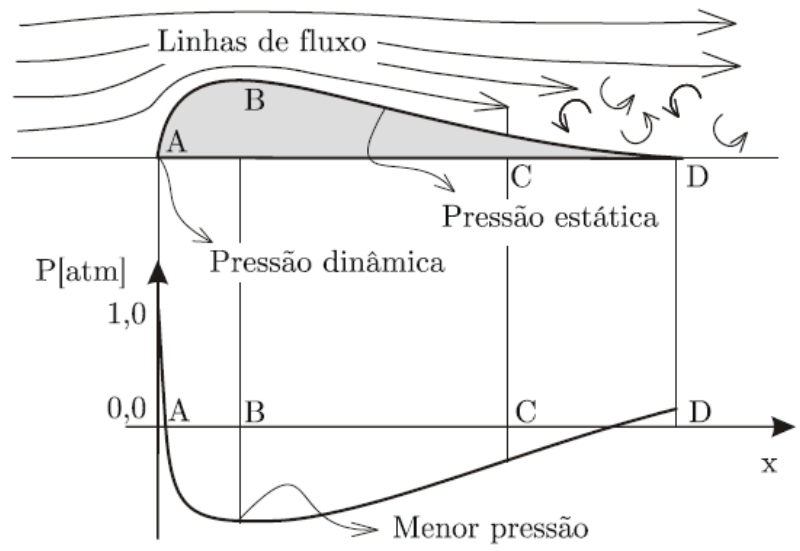

Figura 1 - Escoamento do ar em torno de um corpo Fonte: Leal (2012)

O referido autor explica que quando o ar passa de A para B, adquire maior velocidade, pois diminui a seção de fluxo. Com o aumento da velocidade, a pressão estática do ar diminui e assim, neste trecho, o ar flui sem qualquer problema, pois vai de uma zona de alta pressão para uma zona de baixa pressão. No trecho BD, devido à aceleração sofrida no primeiro trecho e a viscosidade do fluído, as moléculas da camada limite ganham energia. Porém, na parte posterior do corpo há um aumento na seção de fluxo de ar e, assim, uma redução da velocidade, produzindo uma desaceleração da camada limite. As moléculas da camada limite possuem menor energia, sentindo primeiro o efeito da pressão adversa e em um dado ponto do contorno do corpo, a pressão alcança um valor que força o fluxo a voltar em direção a zona de baixa pressão. Leal et al (2012) afirma que a quantidade de ar que retorna aumenta, até a separação da camada limite e, na zona em que o fluxo é reverso, formam-se turbilhões que agitam todo escoamento.

Assim, com essas averiguações percebe-se que a intensidade do atrito de arraste depende de vários fatores, os quais influenciam no comportamento dinâmico do sistema. Então, será proposto o modelo matemático que descreve esse fenômeno com o intuito de proporcionar uma maior captura das características do atrito em questão. 


\section{Modelagem matemática da posição veicular considerando o efeito do atrito de arraste}

Quando um corpo se move através do ar, esse exerce sobre o corpo uma força de resistência que tende a reduzir a sua velocidade, denominada força de arraste. Considerando a figura 2, pode-se observar que essa força de arraste, componente da força aerodinâmica paralela à direção do móvel, depende do atrito viscoso do ar com as superfícies sobre as quais escoa e dos efeitos causados pelo encontro do ar com o corpo em movimento.

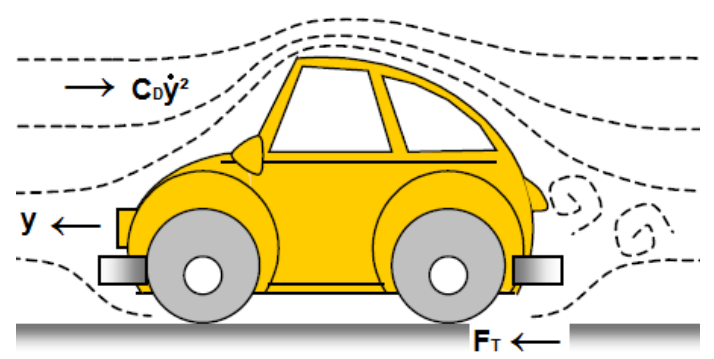

Figura 2 - Desenho esquemático representando o Atrito de Arraste.

Fonte: Os autores.

O modelo não linear de $2^{\mathrm{a}}$ ordem que descreve o comportamento dinâmico da posição veicular é dado pela combinação das equações:

$$
\begin{gathered}
\sum F_{y}=m \cdot \ddot{y} \\
F_{T}-C_{D} \cdot \dot{y}^{2}=m \cdot \ddot{y} \\
m \cdot \ddot{y}+C_{D} \cdot \dot{y}^{2}=F_{T}
\end{gathered}
$$

onde a força de atrito é no sentido contrário ao movimento e é caracterizada pela força de arraste, $-C_{D} \cdot \dot{y}^{2}$, e a força de tração, $F_{T}$, é responsável pelo movimento, logo no sentido positivo adotado. Como posteriormente pretende-se escrever o sistema em variáveis de estado, a equação (3) pode ser favoravelmente escrita como

$$
\ddot{y}+\frac{C_{D}}{m} \cdot \dot{y}^{2}=\frac{F_{T}}{m}
$$

A equação diferencial (4) representa a equação da posição de um veículo considerando os efeitos do atrito de arraste. Nesse sistema, $m$ corresponde à massa total do carro, $C_{D}$ ao coeficiente de atrito viscoso, $y$ à posição inicial, $\dot{y}$ à velocidade inicial, $\ddot{y}$ à aceleração e $F_{T}$ a força de Tração.

A seguir apresenta-se a simulação computacional através da estimação dos parâmetros do modelo matemático, definindo as condições de equilíbrio e avaliando a estabilidade do correspondente sistema.

\section{Simulação computacional do modelo matemático}

Por conveniência, ao usar métodos computacionais para descrever a evolução do sistema, fazse necessário por meio de um processo de linearização em torno de pontos de equilíbrio, transformar a equação diferencial de segunda ordem (4) em duas equações de primeira ordem. Essa técnica utiliza a definição de variáveis de estado, tornando os cálculos mais simples na medida em que possíveis soluções são associadas a determinados parâmetros. Nesse caso, admite-se que para a simulação computacional do sistema, o que se quer não é a forma exata da solução, mas, sim, o seu comportamento dinâmico. 
Inicialmente definem-se as variáveis de estado para determinar os pontos de equilíbrio e a estabilidade no sentido de Lyapunov. Com esses dados obtidos, realiza-se o esboço do plano de fase. Para Slotine e Li (1991), o plano de fase resulta numa família de trajetórias obtidas pelo movimento da dinâmica, em que é possível observar visualmente os efeitos não lineares contidos no estudo qualitativo do sistema.

Assim, considerando $x_{1}=y$ e $x_{2}=\dot{y}$, tem-se a equação (4) em variáveis de estado:

$$
\left\{\begin{array}{c}
\dot{x}_{1}=x_{2} \\
\dot{x}_{2}=-\frac{C_{D}}{m} \cdot x_{2}{ }^{2}+\frac{F_{T}}{m}
\end{array}\right.
$$

É preciso, primeiramente, determinar os pontos de equilíbrio ou pontos críticos. Ponto de equilíbrio é um ponto em que o sistema permanece constante, ou seja, não varia sua posição em relação ao tempo. Os sistemas não lineares freqüentemente apresentam mais de um ponto de equilíbrio.

A solução para o ponto de equilíbrio é dada pelo valor que satisfaz a seguinte relação:

$$
\left\{\begin{array}{l}
\dot{x}_{1}=0 \\
\dot{x}_{2}=0
\end{array}\right.
$$

Ao substituir (6) no sistema (5), nota-se que o único ponto de equilíbrio seria para $x_{2 \theta}=0$ com $F_{T}=0$, onde ambas as variáveis de estado permanecem inalteradas, entretanto em regime permanente ocorre o equilíbrio dinâmico na velocidade de cruzeiro $x_{2 \theta}=\sqrt{\frac{F_{T}}{C_{D}}}$. Chama-se velocidade de cruzeiro ao valor da velocidade em regime permanente, quando a força de atrito de arraste iguala-se a força de tração, a aceleração torna-se nula e a velocidade se estabiliza. A tabela 1 apresenta os valores dos parâmetros utilizados na dinâmica da posição veicular sob o efeito do atrito de arraste.

\begin{tabular}{cccl}
\hline \hline Descrição do parâmetro & Notação & Valores & Observações: \\
\hline Massa do carro & $M$ & $800 \mathrm{~kg}$ & Valor estimado \\
\hline Força de Tração & $F_{T}$ & $3.500 \mathrm{~N}$ & Valor estimado \\
\hline Coeficiente de atrito viscoso & $C_{D}$ & $100 \mathrm{~N} . \mathrm{s} / \mathrm{m}$ & Valor estimado \\
\hline \hline
\end{tabular}

Tabela 1: Parâmetros da dinâmica do atrito veicular

Para esses valores, o sistema apresenta a velocidade de regime permanente de 5,91 m/s.

Conhecendo as soluções de equilíbrio, analisa-se a questão da estabilidade do movimento notando que para qualquer condição inicial adotada, o movimento sempre irá convergir para o equilíbrio dinâmico na velocidade de cruzeiro.

A estabilidade é analisada através da matriz Jacobiana $A$. Assim, o sistema não linear dado pelas equações (5) pode ser linearizado e reescrito na forma matricial $\dot{x}=A x+B u$ tal com:

$$
\left[\begin{array}{l}
\dot{x}_{1}(t) \\
\dot{x}_{2}(t)
\end{array}\right]=\left[\begin{array}{cc}
0 & 1 \\
0 & -\frac{C_{D}}{m}
\end{array}\right]\left[\begin{array}{l}
x_{1}(t) \\
x_{2}(t)
\end{array}\right]+\frac{1}{m}\left[\begin{array}{c}
0 \\
F_{T}
\end{array}\right]
$$


Considerando os valores dos parâmetros da Tabela 1, o determinante da matriz $A$ será nulo.

Nesse caso, trata-se de uma matriz singular e o sistema dinâmico admite infinitas soluções não-nulas. Entretanto, para que exista uma solução não-trivial, é preciso que o determinante da matriz dos coeficientes $(A-\lambda I)$ seja igual a zero:

$$
\operatorname{det}\left(\begin{array}{cc}
0-\lambda & 1 \\
0 & -\left(\frac{C_{D}}{m}+\lambda\right)
\end{array}\right)=0
$$

Resolvendo a equação quadrática, tem-se os autovalores de $A$. De acordo com Slotine e Li (1991), um ponto de equilíbrio é estável se todos os autovalores de $A$ possuam parte real negativa. No presente caso, os autovalores são $\lambda_{1}=0$ e $\lambda_{2}=-0,0625$, logo o sistema não é estável em termos da resposta em posição, pois um dos autovalores é nulo. Isso significa que a posição do móvel apresenta comportamento instável ao variar em função do tempo.

Como $A$ possui um autovalor igual a zero e o outro autovalor real negativo, com um conjunto completo de autovetores linearmente independentes, a origem é considerada um ponto de estabilidade neutra, pois não interfere no sistema. Para o autovalor negativo, o ponto fixo não trivial $x_{2 \varepsilon}=5,91$ é um ponto de equilíbrio da dinâmica da velocidade veicular e é estável.

Essas características podem ser percebidas com o plano de fase do comportamento dinâmico apresentado na figura 3. Para a realização da simulação computacional do modelo matemático proposto, foram adotadas diferentes posições iniciais com suas respectivas velocidades. Ainda, enfatiza-se que a simulação computacional foi realizada com o auxilio da ferramenta MATLAB.

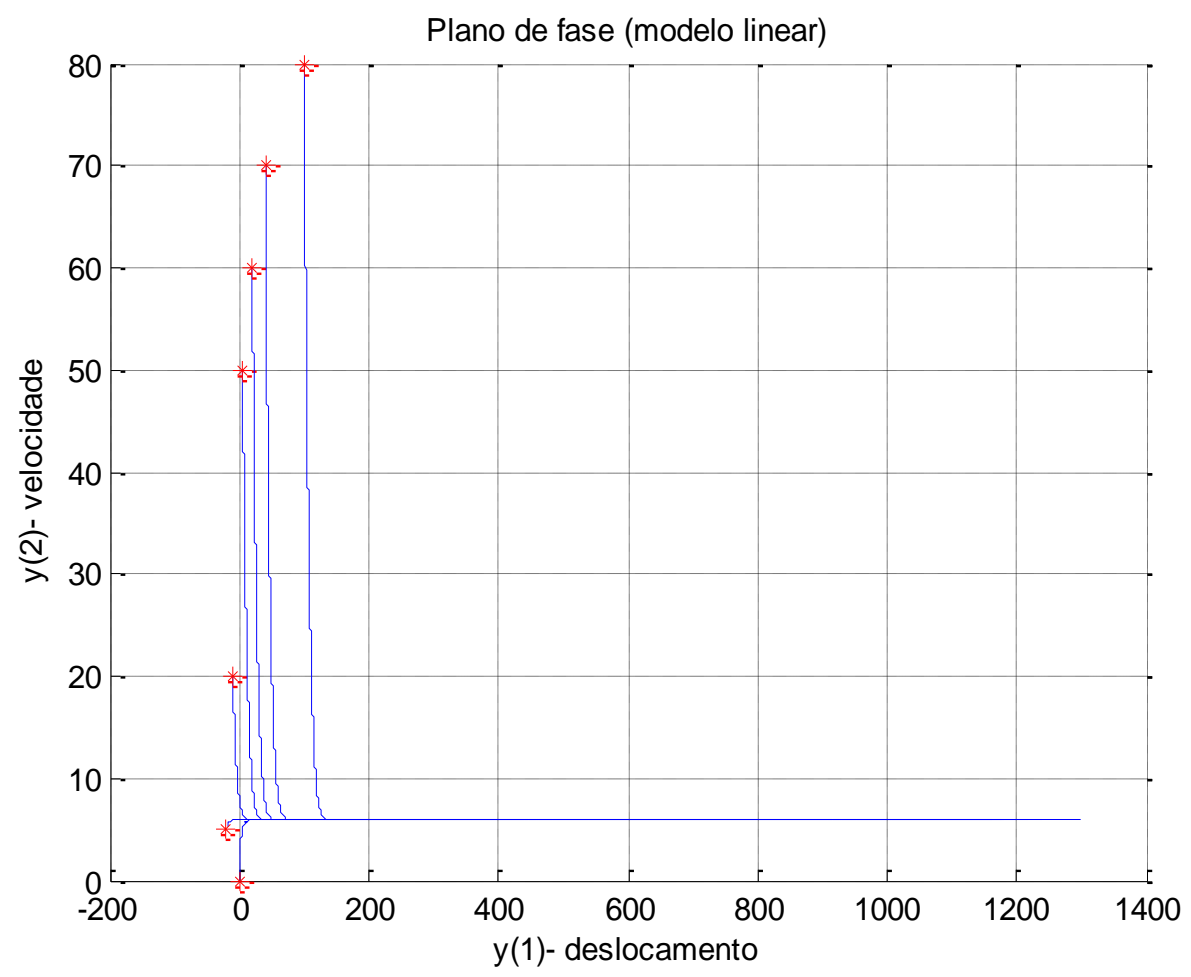

Figura 3 - Comportamento dinâmico da posição veicular considerando o Atrito de Arraste, onde a partir de diferentes condições iniciais (pontos marcados com asterisco e vermelho) todas as trajetórias convergem para o valor da velocidade de cruzeiro, $5,91 \mathrm{~m} / \mathrm{s}$ (linha horizontal).

Fonte: Próprios autores. 


\section{Conclusão}

Apresenta-se a modelagem matemática da dinâmica da posição veicular considerando a não linearidade do atrito de arraste. Através desse estudo, constata-se que a magnitude do atrito de arraste depende do formato aerodinâmico do móvel, da rugosidade e viscosidade do ar, da velocidade imposta, além das diferenças de pressão em torno do veículo. Com o modelo proposto é possível descrever matematicamente o comportamento dinâmico do sistema, incluindo as respectivas interferências. Ao analisar a estabilidade, conclui-se que o sistema não é estável em termos de posição, mas é estável em termos de velocidade veicular, convergindo sempre para velocidade de cruzeiro. Essas verificações são observadas no plano de fase, confirmando que independentemente das condições inicias adotadas, todas as soluções tenderam para o ponto de equilíbrio de velocidade mencionado. Assim, esse trabalho possibilita perceber a importância dos sistemas dinâmicos não lineares para representar diferentes tipos de situações, na evolução temporal.

\section{Referências}

[1] DONADEL, J. Modelagem matemática e controle dos sistemas dinâmicos não-lineares de tempo discreto. 2008. 79 f. Dissertação (Mestrado em Modelagem Matemática) - Universidade Regional do Noroeste do Estado do Rio Grande do Sul, Ijuí, março, 2009.

[2] LEAL, L. C. M., ROSA, E., NICOLAZZI, L. C. Uma introdução à modelagem quase-estática de automóveis Disponível em: http://www.grante.ufsc.br/download/Apostila Ve\%EDculos 2012 Cap1 12.pdf. Acesso em 16 set. 2013.

[3] MIOTTO, F. E. M. Modelagem matemática da dinâmica do atrito e sua aplicação no controle ótimo de um atuador hidráulico. 2009. 110 f. Dissertação (Mestrado em Modelagem Matemática) Universidade Regional do Noroeste do Estado do Rio Grande do Sul, Ijuí, abril, 2009.

[4] MONTEIRO, L. H. A. Sistemas dinâmicos. São Paulo: Editora Livraria da Física, 2002.

[5] SLOTINE, Jean-Jacques E.; LI, Weiping. Applied nonlinear control. Englewood Cliffs: Prentice Hall, 1991.

[6] VALDIERO, A. C. Controle de robôs hidráulicos com compensação de atrito. Disponível em: http://repositorio.ufsc.br/bitstream/handle/123456789/102934/210658pdf?sequence=1. Acesso em 5 set. 2013. 